\title{
REVISÃO INTEGRATIVA SOBRE INSTRUMENTOS DE AVALIAÇÃO DE CONSUMO ALIMENTAR EM CRIANÇAS EM IDADE ESCOLAR
}

\author{
Carla Sílvia Fernandes ${ }^{1}$, Goreti Marques ${ }^{2}$, Constança Festas ${ }^{3}$, Fátima Ferreira ${ }^{4}$, Cristiane Silva ${ }^{5}$
}

\begin{abstract}
RESUMO: A informação sobre o consumo alimentar de uma população permite identificar os sujeitos que se encontram em risco nutricional. O presente estudo teve como objetivos identificar e analisar instrumentos existentes para avaliação do consumo alimentar em crianças em idade escolar. Realizou-se revisão integrativa no período entre 2010 e 2016. Para a análise, segundo os critérios de inclusão estabelecidos, foram selecionados nove artigos. Estes permitiram organizar os resultados em duas áreas temáticas: Instrumentos de avaliação de consumo alimentar, e Limitações da utilização dos instrumentos. O instrumento mais frequentemente utilizado foi o recordatório de 24 horas. Os resultados revelam a necessidade de instrumentos mais abrangentes que permitam fazer face às limitações dos atuais instrumentos em uso.
\end{abstract}

DESCRITORES: Inquéritos alimentares; Crianças; Consumo de alimentos; Comportamento alimentar; Hábitos alimentares.

\section{INTEGRATIVE REVIEW ON DIETARY ASSESSMENT INSTRUMENTS IN SCHOOL-AGE CHILDREN}

ABSTRACT: Information on a population's dietary intake enables the identification of individuals who are at nutritional risk. The objective of the present study was to identify and analyze existing dietary assessment instruments in school-age children. An integrative review was carried out from 2010 to 2016. For analysis, nine articles were selected according to established inclusion criteria. These enabled the organization of the results into two thematic areas: dietary assessment instruments and limitations in the use of instruments. The most used instrument was the 24-hour dietary recall. The results showed the need for more comprehensive instruments in order to deal with limitations of current instruments in use.

DESCRIPTORS: Diet Surveys; Child; Food Consumption; Feeding Behavior.

\section{REVISIÓN INTEGRATIVA SOBRE INSTRUMENTOS DE EVALUACIÓN DE CONSUMO ALIMENTARIO PARA NIÑOS EN EDAD ESCOLAR}

RESUMEN: La información sobre el consumo alimentario de una población permite identificar a los sujetos que se encuentran en riesgo nutricional. El presente estudio tuvo como objetivos identificar y analizar instrumentos existentes para evaluación del consumo alimentario de niños en edad escolar. Se realizó revisión integrativa en el período entre 2010 y 2016 . Para el análisis, según los criterios de inclusión establecidos. Fueron seleccionados nueve artículos. Los mismos permitieron organizar los resultados en dos áreas temáticas: Instrumentos de evaluación de consumo alimentario y Limitaciones a la utilización de los instrumentos. El instrumento utilizado más frecuentemente fue el recordatorio de 24 horas. Los resultados expresan la necesidad de instrumentos más inclusivos que permitan enfrentar las limitaciones de los instrumentos actualmente en uso.

DESCRIPTORES: Encuestas sobre Dietas; Niño; Consumo de Alimentos; Conducta Alimentaria; Hábitos Alimentarios.

${ }^{1}$ Enfermeira. Doutora em Enfermagem. Docente da Universidade Fernando Pessoa. Porto, Portugal

${ }^{2}$ Enfermeira. Doutora em Enfermagem. Docente da Escola Superior de Saúde de Santa Maria. Porto, Portugal.

${ }^{3}$ Enfermeira. Doutora em Enfermagem. Docente da Universidade Católica Portuguesa. Porto, Portugal.

${ }^{4}$ Enfermeira. Mestre em Saúde Pública. Docente da Escola Superior de Saúde de Santa Maria. Porto, Portugal.

${ }^{5}$ Enfermeira. Doutora em Ciências da Saúde. Docente da Escola Superior de Saúde de Santa Maria. Porto Portugal 


\section{INTRODUÇÃO}

A alimentação é importante em qualquer etapa da vida, merecendo especial atenção na infância dada as necessidades nutricionais específicas deste grupo etário ${ }^{(1-2)}$. A obesidade é considerada epidemia mundial, com poucas evidências de que a sua incidência diminuiu ou que tenha atingido um patamar ${ }^{(3)}$. Nos últimos anos, grande volume de pesquisas tem sido realizado a este nível evidenciando determinantes precoces e consequências ${ }^{(2-3)}$. O excesso de peso em idade adulta está associado, em longo prazo, a consequências para a saúde, incluindo hipertensão arterial, hiperglicemia, hipercolesteremia, colelitíase, apneia do sono, e anormalidades ortopédicas ${ }^{(3-4)}$.

Além disso, as crianças com sobrepeso parecem ter menorautoestima ${ }^{(5)}$, associadaao desenvolvimento de problemas sociais e psicológicos, tais como bullying ou outros tipos de discriminação. O bullying baseado no peso está entre as formas mais comuns de assédio moral que os jovens enfrentam. Uma situação que é compartilhada por educadores, pais e estudantes ${ }^{(6)}$.

Com base numa revisão da literatura, existem alguns potenciais marcadores iniciais da obesidade, entre eles a obesidade infantil ${ }^{(3)}$, a qual mostra forte evidência que a intervenção precoce é importante, devendo ser direcionada para apoiar o crescimento e o desenvolvimento saudáveis.

O comportamento e as atitudes, sobretudo em idades mais jovens, nem sempre são consoantes com as boas práticas conhecidas e recomendadas. É certo que a família constitui o alicerce, a solidez e o referencial necessário ao jovem, que passa pelas turbulências identificadas na adolescência e na juventude ${ }^{(2,7)}$. Mas, na sociedade ocidental, o próprio tempo de infância decorre fora desse contexto. É na primeira infância que, progressivamente, as crianças vão se entrecruzando com outros processos educativos e socialmente estruturantes. As escolas começam a assumir papel relevante, por vezes substitutivas da família. Passa a existir o pensar crítico do confronto (quantas vezes em oposição) decorrente do referencial do grupo de pares e de outros modelos adultos emergentes do contexto sócio-ambiental.

Assim, os esforços de intervenção precisam começar em idade precoce, para intervir em prol de alimentação saudável e garantir melhor qualidade e vida, mas é essencial conhecer o indivíduo e sua comunidade. Considerando, no entanto, a emergência da epidemia da obesidade infantojuvenil, enfrentá-la é inadiável, carecendo de conhecê-la, estimando riscos e fazendo as intervenções necessárias. Para isso, é imperativo recolher informações sobre o consumo alimentar, identificando quem se encontra em risco nutricional. Porém, investigar o consumo alimentar de crianças é tarefa desafiadora, existindo controvérsia na literatura sobre o melhor método ${ }^{(8-10)}$.

Cientes desta dificuldade e recorrendo a revisão integrativa da literatura, pretendemos identificar e analisar os instrumentos de avaliação de consumo alimentar, utilizados em crianças em idade escolar, que emergem da produção científica dos últimos anos.

\section{- MÉTODO}

O presente estudo pretendeu agrupar o estado da arte sobre os instrumentos de avaliação nutricional em uso. A revisão integrativa, que apresenta enorme incorporação na pesquisa em enfermagem e na prática baseada em evidência, permite reunir dados de estudos desenvolvidos mediante diferentes metodologias ${ }^{(11)}$. A síntese dos resultados de pesquisas relevantes agiliza a transferência de conhecimento para a prática ${ }^{(12)}$.

Tendo em consideração o conhecimento que se pretendia sintetizar, a revisão teve como ponto de partida a seguinte questão: "Quais os instrumentos de avaliação nutricional utilizados em crianças em idade escolar?"

Para responder à questão, realizou-se pesquisa incorporando as bases de dados bibliográficas Cumulative Index to Nursing \& Allied Health Literature (CINAHL), Medical Literature Analysis and Retrieval System Online (MEDLINE), Nursing \& Allied Health Collection, COCHRANE, MedicLatina, NHS Database, Health Technology Assessments. O rastreio da literatura foi realizado tendo por base a utilização dos descritores: Nutrition Surveys AND feeding behavior AND Child, utilizando-se linguagem 
booleana para o cruzamento dos conjuntos em análise em inglês, português e espanhol.

A pesquisa foi delimitada a título, palavras-chave ou descritores e abstract, com vista a demarcar o conjunto de documentos a rastrear. Após a definição da questão de partida e respetivos descritores, foi definido o protocolo de pesquisa (Figura 1). A pesquisa bibliográfica foi realizada entre maio e junho de 2016.

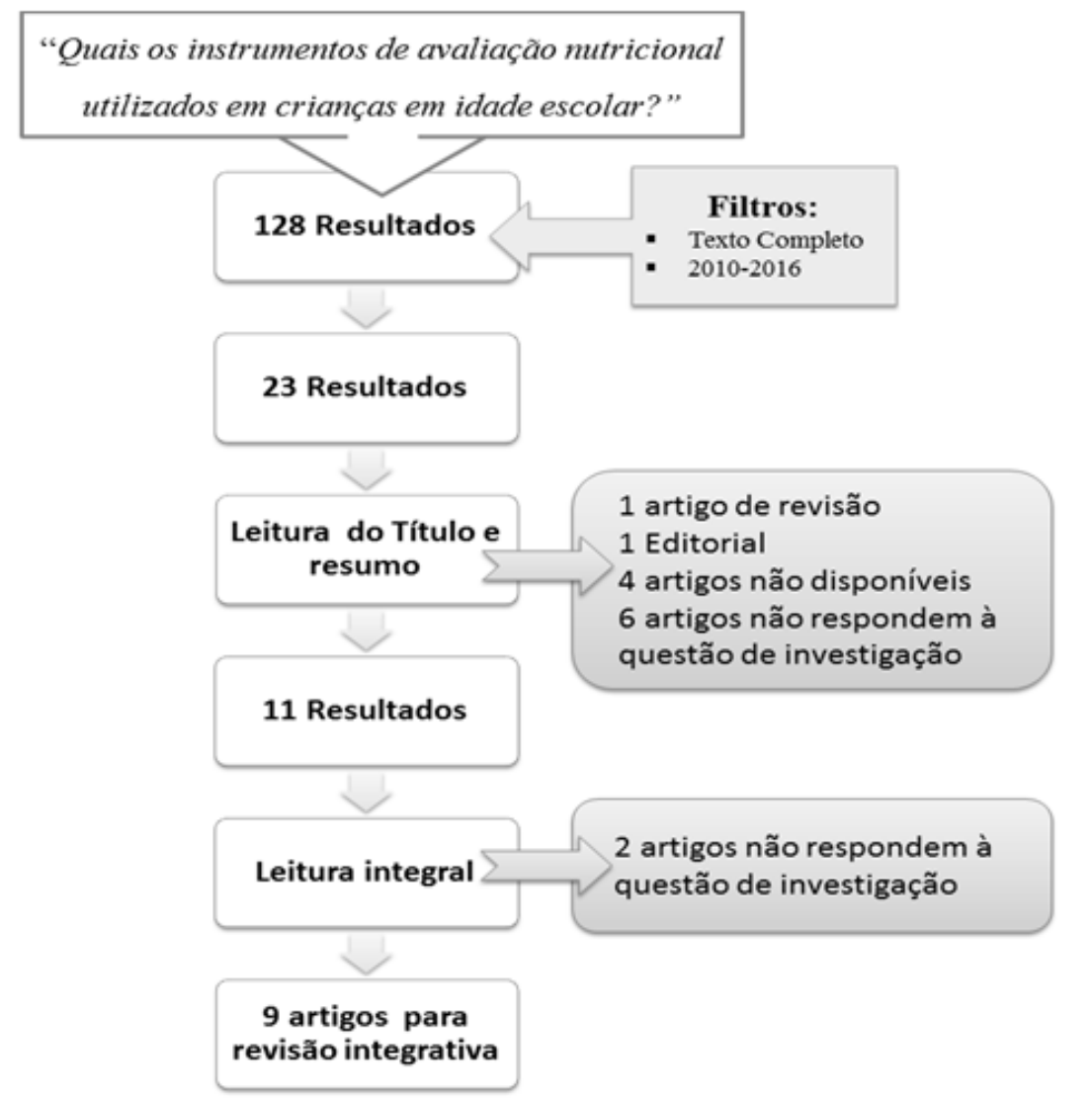

Figura 1 - Protocolo Pesquisa. Porto, PT, 2016

Definiram-se como critérios de inclusão artigos científicos referenciando a utilização de instrumentos de avaliação nutricional, publicados entre os anos de 2010 e 2016. Os critérios de exclusão foram os artigos de revisão, assim como os que não respondiam à questão de pesquisa.

Dentre os 23 artigos identificados, a amostra foi constituída por nove artigos que atenderam aos critérios de inclusão. A análise dos dados é semelhante à de pesquisas convencionais. Esta fase implica em apreciação crítica de cada estudo ${ }^{(12)}$, tendo por base a questão de pesquisa, a razão e a finalidade da obtenção de respostas ${ }^{(11)}$.

\section{RESULTADOS}

Os nove artigos que integraram a amostra são apresentados na Tabela 1 com as seguintes informações: autores, título, ano, periódico e objetivo. 
Tabela 1 - Artigos em análise. Porto, PT, 2016

\begin{tabular}{|c|c|c|c|c|c|}
\hline Código & Autores & Título & Ano & Periódico & Objetivo \\
\hline $\mathrm{A} 1$ & $\begin{array}{l}\text { Popkin B, } \\
\text { Duffey } K^{(13)}\end{array}$ & $\begin{array}{l}\text { Does hunger and satiety } \\
\text { drive eating anymore? } \\
\text { Increasing eating occasions } \\
\text { and decreasing time } \\
\text { between eating occasions }\end{array}$ & 2010 & $\begin{array}{l}\text { The American } \\
\text { Journal Of } \\
\text { Clinical Nutrition }\end{array}$ & $\begin{array}{l}\text { Examinar as tendências de } \\
\text { padronização de refeições }\end{array}$ \\
\hline A2 & $\begin{array}{l}\text { Bevans K, } \\
\text { Sanchez B, } \\
\text { Teneralli R, } \\
\text { Forrest } C^{(14)}\end{array}$ & $\begin{array}{l}\text { Children's eating behavior: } \\
\text { the importance of nutrition } \\
\text { standards for foods in } \\
\text { schools }\end{array}$ & 2011 & $\begin{array}{l}\text { The Journal } \\
\text { Of School Health }\end{array}$ & $\begin{array}{l}\text { Avaliar as contribuições } \\
\text { de ofertas de alimentos e } \\
\text { participação em programas } \\
\text { de almoço escolar sobre o } \\
\text { comportamento alimentar } \\
\text { das crianças. }\end{array}$ \\
\hline A3 & $\begin{array}{l}\text { Kant A, } \\
\text { Graubard B(15) }\end{array}$ & $\begin{array}{l}\text { 20-Year trends in dietary } \\
\text { and meal behaviors were } \\
\text { similar in U.S. children and } \\
\text { adolescents of different } \\
\text { race/ethnicity. }\end{array}$ & 2011 & $\begin{array}{l}\text { The Journal Of } \\
\text { Nutrition }\end{array}$ & $\begin{array}{l}\text { Analisar as mudanças no } \\
\text { consumo alimentar da } \\
\text { população americana, entre } \\
\text { diferentes raças/etnias }\end{array}$ \\
\hline A4 & $\begin{array}{l}\text { Chung A, } \\
\text { Perrin E, } \\
\text { Skinner } A^{(16)}\end{array}$ & $\begin{array}{l}\text { Accuracy of child and } \\
\text { adolescent weight } \\
\text { perceptions and their } \\
\text { relationships to dieting and } \\
\text { exercise behaviors: a } \\
\text { NHANES study }\end{array}$ & 2013 & $\begin{array}{l}\text { Academic } \\
\text { Pediatrics }\end{array}$ & $\begin{array}{l}\text { Analisar como as crianças e } \\
\text { os adolescentes percebem } \\
\text { o seu peso em comparação } \\
\text { com o seu peso real, } \\
\text { associado aos esforços e } \\
\text { comportamentos de perda } \\
\text { de peso. }\end{array}$ \\
\hline A5 & $\begin{array}{l}\text { Madowitz J, } \\
\text { Liang J, } \\
\text { Peterson C, } \\
\text { Rydell S, } \\
\text { Zucker N, } \\
\text { Tanofsky- } \\
\text { Kraff, } \\
\text { Harnack L, et } \\
\text { al }^{(10)}\end{array}$ & $\begin{array}{l}\text { Concurrent and Convergent } \\
\text { Validity of the Eating in } \\
\text { the Absence of Hunger } \\
\text { Questionnaire and } \\
\text { Behavioral Paradigm in } \\
\text { Overweight Children }\end{array}$ & 2014 & $\begin{array}{l}\text { The } \\
\text { International } \\
\text { Journal Of Eating } \\
\text { Disorders }\end{array}$ & $\begin{array}{l}\text { Avaliar a validade } \\
\text { concomitante do } \\
\text { questionário sobre a } \\
\text { alimentação de crianças } \\
\text { (EAH-PC) e auto-relato de } \\
\text { crianças (EAH-C) com o } \\
\text { paradigma comportamental } \\
\text { EAH (EAH\%) e ingestão } \\
\text { alimentar habitual. }\end{array}$ \\
\hline A6 & $\begin{array}{l}\text { Elder J, } \\
\text { Arredondo E, } \\
\text { Campbell } \\
\text { N, Baquero } \\
\text { B, Duerksen } \\
\text { S, Ayala G, } \\
\text { Crespo J, } \\
\text { Slymen D, } \\
\text { McKenzie T(3) }^{\text {McKent }}\end{array}$ & $\begin{array}{l}\text { Individual, family, and } \\
\text { community environmental } \\
\text { correlates of obesity in } \\
\text { Latino elementary school } \\
\text { children }\end{array}$ & 2010 & $\begin{array}{l}\text { The Journal Of } \\
\text { School Health }\end{array}$ & $\begin{array}{l}\text { Analisar os múltiplos níveis } \\
\text { ecológicos no estado de } \\
\text { peso das crianças latinas. }\end{array}$ \\
\hline A7 & $\begin{array}{l}\text { Obeidat } \\
\text { B, Shriver } \\
\text { B, Roman- } \\
\text { Shriver C } C^{(4)}\end{array}$ & $\begin{array}{l}\text { Factors involved in the } \\
\text { persistence of overweight } \\
\text { among children enrolled in } \\
\text { the supplemental } \\
\text { food program for women, } \\
\text { infants, and children. }\end{array}$ & 2010 & $\begin{array}{l}\text { Maternal And } \\
\text { Child Health } \\
\text { Journal }\end{array}$ & $\begin{array}{l}\text { Investigar as diferenças } \\
\text { entre crianças de } 2 \text { a } 5 \\
\text { anos inscritas no Programa } \\
\text { Especial de Nutrição } \\
\text { Suplementar para Mulheres, } \\
\text { Lactentes e Crianças }\end{array}$ \\
\hline A8 & $\begin{array}{l}\text { Niu J, Seo D, } \\
\text { Lohrmann } \\
D^{(17)}\end{array}$ & $\begin{array}{l}\text { Weight perception and } \\
\text { dietary intake among } \\
\text { Chinese youth, 2004-2009. }\end{array}$ & 2014 & $\begin{array}{l}\text { International } \\
\text { Journal Of } \\
\text { Behavioral } \\
\text { Medicine }\end{array}$ & $\begin{array}{l}\text { Investigar a dimensão e } \\
\text { correlação da percepção } \\
\text { errada do peso a ingestão } \\
\text { dietética entre os jovens } \\
\text { chineses. }\end{array}$ \\
\hline A9 & $\begin{array}{l}\text { Affenito S, } \\
\text { Thompson } \\
\text { D, Dorazio } \\
\text { A, Albertson } \\
\text { A, Loew A, } \\
\text { Holschuh } \\
\mathrm{N}^{(18)}\end{array}$ & $\begin{array}{l}\text { Ready-to-eat cereal } \\
\text { consumption and the } \\
\text { School Breakfast Program: } \\
\text { Relationship to nutrient } \\
\text { intake and weight. }\end{array}$ & 2013 & $\begin{array}{l}\text { The Journal Of } \\
\text { School Health }\end{array}$ & $\begin{array}{l}\text { Analisar a associação do } \\
\text { consumo de cereais de café } \\
\text { da manhã pronto-a-comer } \\
\text { com a ingestão de nutrientes } \\
\text { e o peso dos estudantes }\end{array}$ \\
\hline
\end{tabular}


Enquadrada na delimitação temporal entre 2010 e 2016, os artigos analisados apresentam a distribuição apresentada na Figura 2.

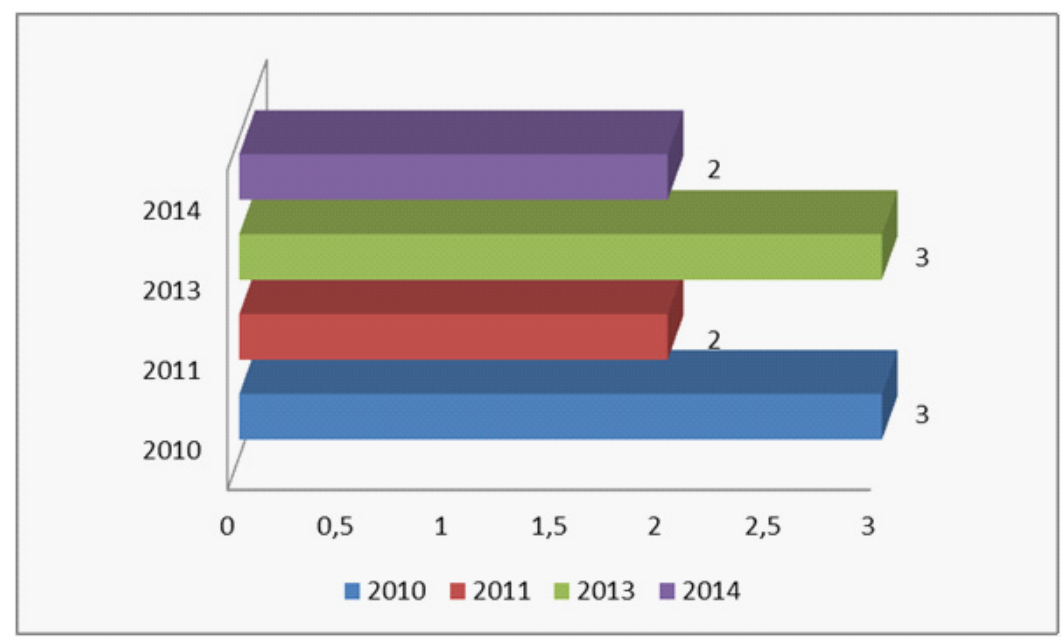

Figura 2 - Distribuição dos estudos, segundo o ano de publicação. Porto, PT, 2016

Os resultados apresentam maior concentração das produções no ano de 2010 e 2013. Salienta-se que os artigos encontrados em 2015 e 2016 não respondiam à questão de pesquisa. Em relação ao idioma de publicação, todos eles são publicados em inglês. No que se refere ao local de realização do estudo, a maioria ( $\mathrm{n}=$ oito) foi realizada nos Estados Unidos e apenas um foi realizado na China.

\section{- DISCUSSÃO}

Os estudos analisados foram categorizados em duas áreas: instrumentos de avaliação de consumo alimentar e limitações dos instrumentos, que conduziram a discussão com a finalidade de dar resposta ao questionamento feito.

\section{Instrumentos de avaliação de consumo alimentar}

A avaliação de consumo alimentar na prática clínica é realizada com a finalidade de fornecer subsídios para o desenvolvimento e a implantação de planos nutricionais ${ }^{(9)}$. A medida correta de uma dieta constitui-se tarefa extremamente complexa e desafiadora para um epidemiologista nutricional, e, tratando-se de crianças e adolescentes, a tarefa torna-se mais complexa e de grande preocupação ${ }^{(19)}$. Nos artigos analisados, são relatados a utilização de recordatório de 24 horas, os questionários de frequência alimentar e o diário alimentar.

\section{Recordatório de 24 horas}

O recordatório de 24 horas consiste em obter informações escritas ou verbais sobre a ingestão alimentar de um dia ${ }^{(19)}$. Este compreende a definição e a quantificação de todos os alimentos e bebidas ingeridas no período anterior à entrevista, que podem ser as 24 horas precedentes ou, sendo o mais comum, o dia anterior ${ }^{(9)}$.

Na pesquisa realizada no estudo A1, que pretendia examinar as tendências de padronização de refeições, por meio de estudo transversal em crianças dos 2 aos 8 anos de idade, os autores utilizaram em três momentos o recordatório alimentar de 24 horas. No primeiro momento, utilizaram o The Nationwide Food Consumption Survey de 1977-1978 que obtem informações sobre todos os alimentos consumidos em casa e fora de casa durante três dias consecutivos, usando um único entrevistador ${ }^{(13)}$. 
Na segunda fase, utilizaram o Continuing Survey of Food Intakes by Individuals CSFII de 1994-1998 com dados recolhidos, por meio de entrevistas efetuadas em 2 dias não consecutivos (espaçados entre 3-10 dias). E por último, os dados do NHANES-National Heath and Nutrition Examination Survey de 2003-2006 que incluiu 2 dias não consecutivos de coleta de dados dietéticos de 24 horas. No $1^{\circ}$ dia as entrevistas foram conduzidas por entrevistadores dietéticos treinados numa Unidade Móvel, enquanto as entrevistas do dia 2 foram realizadas por telefone 3-10 dias após a $1^{\text {a }}$ entrevista $^{(13)}$.

No estudo A3, cujo objetivo era analisar as mudanças no consumo alimentar da população americana entre diferentes raças/etnias, por meio de estudo transversal numa população entre os 2 e 19 anos, também foram utilizados os dados do NHANES de 1988-1994, 1999-2002 e 2003-2006 de dados dietéticos de 24 horas $^{(15)}$. Outros autores (A4) recorreram também ao NHANES, cujo objetivo era analisar como as crianças e os adolescentes percebem o seu peso em comparação com o seu peso real, numa população de crianças entre os 8 e 15 anos $^{(16)}$.

No estudo A5, os autores utilizaram, entre outros instrumentos, o recordatório de 24 horas, aplicadas em três momentos não consecutivos, conduzidos por um entrevistador dietético treinado, numa população entre os 8 e 12 anos $^{(10)}$.

O estudo A9 teve a finalidade de analisar a associação do consumo de cereais de café da manhã com a ingestão de nutrientes e o peso dos estudantes, entre os 5 e 18 anos. Os dados foram retirados do Third School Nutrition Dietary Assessment Study (SNDA-III) de 2004 a 2005 que realizaram um recordatório, acompanhado de entrevista com os pais ${ }^{(18)}$.

\section{Questionário de frequência de consumo alimentar (QFCA)}

O QFCA tem como principal objetivo conhecer o consumo habitual de determinado grupo populacional, e foi desenvolvido para obter informação sobre o padrão alimentar e a ingestão de alimentos ou nutrientes específicos ${ }^{(19)}$. É também importante avaliar a frequência de consumo de determinados alimentos, tanto daqueles que, se consumidos em excesso, podem comprometer a qualidade da dieta e o estado de saúde, quanto daqueles que são fonte de nutrientes. Neste caso é solicitado, a partir de uma lista de alimentos, a informação sobre a frequência de consumo de cada item $^{(9)}$.

No que se refere às análises, no estudo A2, o objetivo foi avaliar as contribuições de ofertas de alimentos e participação em programas de almoço escolar, relacionando-os com o comportamento alimentar das crianças. Os autores utilizaram entre outros instrumentos o Student Eating Behavior. A escala é composta por oito itens para avaliar os comportamentos alimentares positivos e negativos, assim como três itens adicionais referentes ao consumo de batatas fritas, doces e fast food ${ }^{(14)}$.

Num estudo já referido anteriormente (A5), sobre validade de um questionário de alimentação na ausência de fome e comportamento de crianças com sobrepeso, acrescido do recordatório de 24 horas, foram utilizados outros dois questionários: The Eating in the Absence of Hunger Questionnaire for Children and Adolescents (EAH-C) e The Eating in the Absence of Hunger Questionnaire for Children and Adolescents Parent Report of Child (EAH-PC). Ambos possuem 14 perguntas, divididas em três subescalas: "afetividade negativa", "alimentação extra", "fadiga/tédio"; o EAH-C é preenchido pela criança ou adolescente, enquanto o EAH-PC é preenchido pelos pais ${ }^{(10)}$.

No estudo realizado em crianças de 2 a 5 anos (A7), a ingestão alimentar da criança foi avaliada por meio de um questionário de frequência alimentar de 29 itens, nominado Food Frequency Questionnaire (FFQ). Foram utilizadas duas listas separadas de frequências alimentares, uma para avaliar o consumo diário de alimentos comuns e outra para avaliar o consumo semanal de outros alimentos $^{(4)}$. Este instrumento também foi utilizado no estudo A6, mas neste caso preenchido pelos pais, numa população de crianças com idades compreendidas entre os 5 e 7 anos. Ao Food Frequency Questionnaire (FFQ) foram adicionados alguns alimentos considerados obesogênicos, avaliando a frequência da sua ingestão(3). 
Esse método consiste em anotar em formulários previamente estruturados todos os alimentos e bebidas consumidas e suas respetivas quantidades durante determinado período, em geral ao longo de um dia $^{(19)}$, podendo ser aplicado ao longo de três, cinco ou sete dias ${ }^{(9)}$. O método poderá ser mais completo e preciso recorrendo ao uso de balança ou a ajuda de diferentes tamanhos de porções ou representações do que foi consumido em medidas caseiras tradicionalmente usadas ${ }^{(9)}$.

Da mesma forma que o diário alimentar de 24 horas, o registro alimentar recolhe informações quando a criança ou pessoa responsável registra, em formulários especialmente desenhados, todos os alimentos e bebidos consumidos ${ }^{(19)}$.

O estudo A8 é o único com referência a este instrumento, cujo objetivo era investigar a dimensão e correlação da percepção errada do peso e a ingestão dietética entre os jovens chineses, utilizando o registo de ingestão alimentar em três dias consecutivos ${ }^{(17)}$.

\section{Limitações dos instrumentos}

Embora nem sempre explorado ao longo dos artigos em análise, a avaliação do consumo alimentar tem um papel crítico na área de pesquisa e também no desenvolvimento de programas, pois cada instrumento apresenta vantagens e desvantagens com a sua aplicação. As pesquisas de consumo alimentar utilizam instrumentos eficazes e de baixo custo, no entanto devido ao uso e as limitações de cada método, a escolha do instrumento para medir a informação dietética não constitui tarefa fácil(19).

Em alguns estudos em análise são evidenciadas algumas limitações com a utilização de alguns instrumentos. No estudo A1 os autores salientam que o autorelato da ingestão alimentar, com base em recordações de 24 horas, pode não ser representativo do consumo habitual de um indivíduo. Além de que existe uma subnotificação dos alimentos que se pensa estarem ligados à obesidade, como a gordura e o açúcar ${ }^{(13)}$. Esta informação é validada por outros autores no que se refere ao recordatório de 24 horas $^{(13,18)}$.

Devido à variabilidade individual da ingestão de alimentos, as estimativas de uma única recordação de 24 horas não são apropriadas para a estimativa das ingestões usuais de indivíduos. Outra preocupação com a medição dietética diz respeito aos erros de notificação dietética, especialmente a subnotificação da ingestão de certos alimentos ${ }^{(15)}$.

Da mesma forma, com aplicação do questionário de frequência alimentar no estudo A2 e A7, os autores referem que apesar de as crianças serem geralmente repórteres confiáveis e precisos de seus comportamentos alimentares, podem ser considerados vieses nestas informações ${ }^{(14,16)}$. Outro aspeto salientado em relação ao questionário de frequência alimentar era a necessidade de integrar informações sobre o tamanho das porções ${ }^{(4)}$.

Apesar de não referenciados pelos autores, cada um dos métodos tem vantagens quando aplicado em crianças ${ }^{(9,19)}$. Os instrumentos mais apropriados, quando se deseja quantificar e avaliar a ingestão de nutrientes, são aqueles que são capazes de recolher a informação detalhada sobre o consumo, ou seja, alimentos consumidos e as quantidades ingeridas. Neste caso, os métodos mais utilizados são o recordatório de 24 horas e o diário alimentar ${ }^{(9)}$.

\section{CONCLUSÃO}

Medir o consumo alimentar constitui uma tarefa complexa e desafiadora, principalmente em crianças. Apesar da complexidade da avaliação do consumo alimentar, e considerando as vantagens e limitações dos instrumentos, esta deve fazer parte dos programas de alimentação nutricional. A avaliação do consumo alimentar requer a definição clara dos objetivos a serem alcançados para orientar a seleção dos instrumentos, incluindo diversos fatores como o tipo de dieta, idade, cultura, crenças, meio social e econômico, entre outros.

O presente estudo pretendeu agrupar o conhecimento existente sobre a utilização dos Instrumentos de Avaliação do Consumo Alimentar, por meio de uma revisão integrativa. A análise da evidência científica obtida permitiu organizar os resultados em duas áreas temáticas: Instrumentos de avaliação 
de consumo alimentar, e Limitações da utilização dos instrumentos.

O instrumento mais frequentemente utilizado nos estudos foi o recordatório de 24 horas, sendo referida também a utilização do questionário de frequência alimentar e o diário alimentar. Embora omisso nos estudos em análise, o diário alimentar é referido na literatura como apresentando menos desvantagens, considerado o método mais válido para medir a ingestão alimentar, não devendo ultrapassar os sete dias.

Os resultados revelam a necessidade de maior desenvolvimento a este nível que permitam fazer face às limitações dos instrumentos atuais em uso.

\section{REFERÊNCIAS}

1. Portugal. Direção-Geral da Saúde. Programa Nacional para a Promoção da Alimentação Saudável. Portugal Alimentação Saudável em números - 2014. Lisboa: DGS; 2014.

2. Elder JP, Arredondo EM, Campbell N, Baquero B, Duerksen S, Guadalupe A, et al. Individual, family, and community environmental correlates of obesity in Latino elementary school children. JOSH. [Internet] 2010;80(1) [acesso em 25 nov 2016]. Disponível: http://dx.doi.org/10.1111/j.1746-1561.2009.00462.x.

3. Brisbois TD, Farmer AP, McCargar LJ. Early markers of adult obesity: a review. Obes Rev. [Internet] 2012;13(4) [acesso em 25 nov 2016]. Disponível: http://dx.doi.org/ 10.1111/j.1467-789X.2011.00965.x.

4. Obeidat BA, Shriver BJ, Roman-Shriver CR. Factors involved in the persistence of overweight among children enrolled in the supplemental food program for women, infants, and children. Matern Child Health J. [Internet] 2010;14(2) [acesso em 23 nov 2016]. Disponivel: http://dx.doi.org/10.1007/s10995-009-0457-2.

5. Lee PY, Cheah WI, Chang CT, Siti Raudzah G. Childhood obesity, self-esteem and health-related quality of life among urban primary schools children in Kuching, Sarawak, Malaysia. Malays J Nutr. 2012;18(2):207-19.

6. Puhl R, Luedicke J, King KM. Combating weight-based bullying in schools: is there public support for the use of litigation?. J Sch Health. [Internet] 2015;85(6) [acesso em 25 nov 2016]. Disponível: http://dx.doi.org/10.1111/ josh.12264.

7. Collins C, Duncanson K, Burrows T. A systematic review investigating associations between parenting style and child feeding behaviours. J Hum Nutr Diet. [Internet] 2014;27(6) [acesso em 25 nov 2016]. Disponível: http:// dx.doi.org/10.1111/jhn.12192.

8. Consolmagno DC, Assunção NA, Giovannetti TL, Zeraib DP, Hinnig PF, Freaza SRM, et al. Treinamento de escolares de 7 a 10 anos para o preenchimento de um Diário Alimentar. Rev. bras. epidemiol. [Internet] 2009;12(3) [acesso em 19 jun 2016]. Disponível: http://dx.doi.org/10.1590/S1415-790X2009000300009.

9. Fisberg RM, Marchioni DML, Colucci ACA. Avaliação do consumo alimentar e da ingestão de nutrientes na prática clínica. Arq Bras Endocrinol Metab. [Internet] 2009;53(5) [acesso em 19 jun 2016]. Disponivel: http://dx.doi. org/10.1590/S0004-27302009000500014.

10. Madowitz J, Liang J, Peterson CB, Rydell S, Zucker NL, Tanofsky-Kraff M, et al. Concurrent and convergent validity of the eating in the absence of hunger questionnaire and behavioral paradigm in overweight children. Int J Eat Disord. [Internet] 2014;47(3) [acesso em 25 nov 2016]. Disponível: http://dx.doi.org/10.1002/eat.22213.

11. Soares CB, Hoga LAK, Peduzzi M, Sangaleti C, Yonekura T, Silva DRAD. Revisão integrativa: conceitos e métodos utilizados na enfermagem. Rev. esc. enferm. USP. [Internet] 2014;48(2) [acesso em 25 nov 2016]. Disponível: http://dx.doi.org/10.1590/S0080-6234201400002000020.

12. Whittemore R, Knafl K. The integrative review: updated methodology. J Adv Nurs. [Internet] 2005;52(5) [acesso em 24 abr 2016]. Disponível: http://dx.doi.org/10.1111/j.1365-2648.2005.03621.x.

13. Popkin BM, Duffey KJ. Does hunger and satiety drive eating anymore? Increasing eating occasions and decreasing time between eating occasions in the United States. Am J Clin Nutr. [Internet] 2010;91(5) [acesso em 23 nov 2016]. Disponível: http://dx.doi.org/10.3945/ajcn.2009.28962. 
14. Bevans KB, Sanchez B, Teneralli R, Forrest CB. Children's eating behavior: the importance of nutrition standards for foods in schools. J Sch Health. [Internet] 2011;81(7) [acesso em 23 nov 2016]. Disponível: http:// dx.doi.org/10.1111/j.1746-1561.2011.00611.x.

15. Kant AK, Graubard BI. 20-Year trends in dietary and meal behaviors were similar in U.S. children and adolescents of different race/ethnicity. J Nutr. [Internet] 2011;141(10) [acesso em 23 nov 2016]. Disponível: http:// dx.doi.org/10.3945/jn.111.144915.

16. Chung AE, Perrin EM, Skinner AC. Accuracy of child and adolescent weight perceptions and their relationships to dieting and exercise behaviors: a NHANES study. Acad Pediatr. [Internet] 2013;13(4) [acesso em 23 nov 2016]. Disponível: http://dx.doi.org/10.1016/j.acap.2013.04.011.

17. Niu J, Seo DC, Lohrmann DK. Weight perception and dietary intake among Chinese youth, 2004-2009. Int J Behav Med. [Internet] 2014;21(4) [acesso em 23 nov 2016]. Disponível: http://dx.doi.org/10.1007/s12529-0139332-z.

18. Affenito SG, Thompson D, Dorazio A, Albertson AM, Loew A, Holschuh NM. Ready-to-eat cereal consumption and the School Breakfast Program: relationship to nutrient intake and weight. J Sch Health. [Internet] 2013;83(1) [acesso em 23 nov 2016]. Disponível: http://dx.doi.org/10.1111/j.1746-1561.2012.00744.x.

19. Cavalcante AAM, Priore SE, Franceschini SCC. Estudos de consumo alimentar: aspectos metodológicos gerais e o seu emprego na avaliação de crianças e adolescentes. Rev. Bras. Saude Mater. Infant. [Internet] 2004;4(3) [acesso em 26 nov 2016]. Disponível: http://dx.doi.org/10.1590/S1519-38292004000300002. 